\title{
Chloroforme et signification : pourquoi la littérature est-elle si soporifique à l'école?
}

Jérôme David

\section{(2) OpenEdition \\ 12 Journals}

Édition électronique

URL : http://journals.openedition.org/edl/604

DOI : $10.4000 /$ edl. 604

ISSN : 2296-5084

Éditeur

Université de Lausanne

\section{Édition imprimée}

Date de publication : 15 mars 2014

Pagination : 19-32

ISBN : 978-2-940331-34-5

ISSN : 0014-2026

Référence électronique

Jérôme David, "Chloroforme et signification : pourquoi la littérature est-elle si soporifique à l'école? », Études de lettres [En ligne], 1 | 2014, mis en ligne le 15 mars 2017, consulté le 20 décembre 2020. URL: http://journals.openedition.org/edl/604 ; DOI : https://doi.org/10.4000/edl.604 


\section{CHLOROFORME ET SIGNIFICATION : POURQUOI LA LITTÉRATURE EST-ELLE SI SOPORIFIQUE À L'ÉCOLE?}

Trois essais contemporains débouchent sur un constat commun : l'impossibilité, pour les œuvres littéraires lues par les élèves, à faire sens au-delà de la classe. Les présupposés des savoirs critiques transposés à l'école depuis quarante ans expliquent cette impossibilité, puisqu'ils font du "second degré» de la littérature le critère de la valeur artistique et le signe d'élection des lecteurs avertis. Or ce «second degré» s'accompagne d'une forme d'expérience esthétique souvent hors de portée des élèves; il impose surtout un mode de subjectivation par la littérature dont les mots d'ordre sont la défiance, le scepticisme et l'ironie solitaire. Le "premier degré de la littérature" proposé ici se présente comme une alternative possible à cette conception.

Celui qui lit sérieusement renonce, durant sa lecture, à juger; pour juger, il faudrait se tenir à distance et au-dehors, réduire l'œuvre à l'état d'objet, d'organisme inerte. Le lecteur pénétrant s'installe dans l'œuvre pour épouser les mouvements d'une imagination et les dessins d'une composition; il est trop occupé à participer pour se reprendre, à vivre une aventure d'être pour se poser en spectateur. Comment jugerait-il ce dont il se rend si intimement complice?

Jean Rousset ${ }^{1}$

Il existe aujourd'hui trois diagnostics concurrents de la crise déclarée de l'enseignement de la littérature.

I. J. Rousset, Forme et signification, p. XIV. 
Le premier reproche au formalisme des approches privilégiées en classe, et à la technicité des programmes d'études, de faire écran à la leçon des œuvres littéraires: au lieu d'inviter les élèves à réfléchir sur leur condition d'adolescents européens du XXI ${ }^{\mathrm{e}}$ siècle, l'attention très analytique aux textes n'exerce, pour ses détracteurs, qu'une compétence aberrante: celle qui consiste à repérer dans la littérature non pas ce que les textes pourraient dire aux élèves, mais ce que les consignes scolaires demandent d'y reconnaître, soit un catalogue de notions assimilées à tort à un savoir interprétatif ou historique quelconque.

C'est la position défendue par Tzvetan Todorov dans La littérature en péril. Pour lui, le formalisme étouffe l'humanisme qu'enseignent d'euxmêmes les chefs-d'œuvre. L'école, en favorisant un rapport techniciste aux textes, demande aux élèves de regarder le doigt de l'enseignant plutôt que la lune d'un patrimoine culturel précieux, où gît une connaissance plurimillénaire de la nature humaine en attente d'être découverte et réactualisée. Les élèves sont alors coupés de ce qui, dans la littérature, serait le plus à même de les toucher et de les passionner. Ils ne voient plus dans la fréquentation des œuvres littéraires à l'école qu'un exercice vide de sens, dont l'inutilité se confond alors pour eux avec l'inanité de la lecture même des ouvres littéraires, en classe ou ailleurs.

Cette position réagit à la réforme de l'enseignement instaurée en France à la fin des années 1990 : le «formalisme» que dénonce Todorov est selon lui un effet des nouveaux programmes; un formalisme d'ingénieur, donc, et non d'interprète.

Un tel formalisme correspondait toutefois, dans l'esprit des concepteurs de cette réforme, à une sorte de rationalisation démocratique de l'interprétation des textes littéraires. Ce qui a présidé au remaniement des programmes français du secondaire il y a une quinzaine d'années, c'est d'abord un parti pris égalitariste visant à entraver la reproduction des inégalités sociales par l'école - reproduction subreptice et implacable des atouts et des handicaps sociaux dont les premiers travaux sociologiques de Pierre Bourdieu et de Jean-Claude Passeron avaient dressé le constat critique trente ans plus tôt ${ }^{2}$.

2. Voir notamment, parmi leurs nombreuses publications individuelles et collectives sur la question dans les années 1960, les deux ouvrages centraux de Pierre Bourdieu et Jean-Claude Passeron: Les Héritiers et La Reproduction. 
L'explicitation des compétences attendues de l'élève, l'inventaire des notions à acquérir, la spécification méticuleuse des étapes de l'interprétation littéraire - tout cela avait pour but de porter au jour l'implicite des exigences scolaires et de mettre sur un pied d'égalité les élèves d'origine bourgeoise, déjà familiers de l'école et de la littérature, et les élèves issus de milieux moins favorisés sur le plan culturel, qui n’auraient jamais vu de livre à la maison, n'auraient jamais entendu parler à table de Balzac, de Zola ou de Sartre et n'auraient donc aucune idée ni de l'histoire littéraire, ni des manières dont on peut parler d'un texte.

Le diagnostic de Todorov est indissociable de ces débats. Il est très situé. Pour autant, on le retrouve aujourd'hui invoqué ailleurs qu'en France. La formalisation méticuleuse des contenus d'apprentissage est, aux yeux de celles et ceux qui partagent l'avis de Todorov, à l'origine à la fois d'une crise de la vocation chez les enseignants et d'une crise de la motivation chez les élèves.

Le second diagnostic en appelle à une autre hypothèse: l'enseignement de la littérature pâtit aujourd'hui d'une dérive des études littéraires. Ce n'est pas la lecture effective des œuvres littéraires qui est en cause, puisqu'il ne s'est jamais autant vendu de romans ou de poésie dans toute l'histoire de l'humanité, et notamment dans le secteur de la littérature pour la jeunesse. Le problème est ailleurs: dans l'évolution des études littéraires au cours des vingt dernières années. C'est la position de JeanMarie Schaeffer dans sa Petite écologie des études littéraires. Pourquoi et comment étudier la littérature?

Il convient, selon lui, de rompre avec certaines routines savantes de l'interprétation littéraire aussi bien qu'avec leurs avatars scolaires. Les études littéraires ont hérité, nous dit-il, d'une conception de la littérature indissociable d'une revendication des écrivains à l'autonomie de leur activité - la Littérature, avec majuscule, y a revêtu les dehors d'une exception culturelle et symbolique, jugée sans rapport avec les autres formes de discours.

On pourrait ajouter dans son sillage que les catégories savantes élaborées pour accompagner et consolider cette revendication à l'autonomie ont tendu à prouver cette exception de la littérature par une série de démarcations et de restrictions: un texte littéraire n'a pas de référent; il ne prétend pas à la vérité; il parle du langage plutôt que du monde; il parle de lui-même plutôt que du langage; la lecture 
référentielle est une illusion, une naïveté et un traquenard; les textes dialoguent entre eux exclusivement, dans un intertexte infini; ils n'ont qu'un rapport critique et démystificateur, sinon hautain et condescendant, avec les discours de la science ou du sens commun; etc. On aura reconnu dans cet inventaire l'illusion référentielle de Michael Riffaterre, la fonction poétique de Roman Jakobson, l'écriture intransitive de Roland Barthes, l'intertextualité de Julia Kristeva ou le courant récent de la sociocritique. Autant de notions encore largement en usage de nos jours.

Schaeffer, au contraire, récuse cette exception de la littérature. Il rapproche les œuvres littéraires d'autres activités dont les études littéraires n'ont jusqu'à présent pas tenu compte. En abordant la littérature sous l'angle de la fiction, et en envisageant la fiction en un sens élargi et appréciatif (c'est-à-dire sans y voir l'ensemble des discours qui feind raient de dire la vérité et asserteraient le faux), Schaeffer a ouvert la voie à un double renouvellement: (i) l'examen des univers dans lesquels les lecteurs s'immergent lorsqu'ils suivent les consignes des œuvres (des univers dont l'existence est rendue possible par le langage, certes, mais qui n'existent pas seulement dans le langage); et (ii) l'intégration de la littérature dans un ensemble d'activités de simulation virtuelle dont la fiction n'est que l'une des variantes et où elle côtoie, par exemple, les jeux (et notamment les jeux vidéo).

Le diagnostic de l'enseignement qu'établit Schaeffer est, on le voit, le pendant de ses propositions théoriques. En ce sens, il décrit une crise dans l'enseignement dont la résolution serait déjà amorcée dans les études littéraires.

Le troisième diagnostic consiste à attribuer la crise de l'enseignement de la littérature au fait que les élèves se voient le plus souvent assigner un rôle passif face aux textes abordés en classe. Ou, plus précisément, se voient retirer toute possibilité de peser sur les processus scolaires d'analyse et d'interprétation de la littérature.

C'est, entre les lignes, la position d'Yves Citton dans Lire, interpréter, actualiser. Pourquoi les études littéraires?3. Aussi ce dernier propose-t-il de transformer l'analyse de textes en classe en laboratoire démocratique, d'en faire l'occasion d'un apprentissage collectif des règles du vivre-

3. Voir plus particulièrement le chapitre X («Scolarisation»). 
ensemble. Au lieu que l'enseignant fixe les questions auxquelles devront répondre ses élèves, Citton préconise de faire en sorte que les élèves soient amenés à poser eux-mêmes aux textes étudiés les questions qui leur semblent pertinentes. La classe devient alors un collectif en formation; les élèves, dans leur négociation de ce qui fait sens dans un texte, s'instaurent en communauté interprétative; et chacun d'eux, dans cette série d'échanges autour d'une ouvre littéraire, éprouve sa propre puissance herméneutique.

Des dispositifs pédagogiques existent déjà, on le sait, qui favorisent ce genre de délégation interprétative aux élèves. Les cercles de lecture, par exemple ${ }^{4}$. Rassemblés en petits groupes, les élèves y délibèrent de ce qu'il leur importe de souligner dans un passage donné, extrait d'une œuvre littéraire, puis ils font part, de façon argumentée, de leur discussion au reste de la classe qui en débat à son tour.

Les réflexions de Citton ne vont pas jusqu'à ce degré de détail didactique. Elles ne soulèvent donc pas le problème épineux des éventuels conflits d'interprétation qui pourraient surgir au sein de la classe: un différend exégétique entre les élèves doit-il être résorbé à tout prix, sans quoi la rationalité de l'analyse s'en trouverait menacée - ou cette pluralité des significations du texte est-elle au contraire l'indice de sa dimension esthétique? Citton n'aborde pas non plus la question redoutable du statut forcément inégal de l'enseignant dans cette communauté d'interprétation démocratique: est-il l'équivalent d'un élu qui aurait récolté 99,4\% des voix de ses élèves - ou incarne-t-il une sorte de constitution scolaire dont les principes généraux (explicitation du raisonnement, pertinence des preuves, cohésion argumentative, etc.) serviraient de cadre aux disputes menées en classe? Ce qui m'intéresse cependant ici, c'est avant tout cette hypothèse d'une passivité des élèves dans la transmission scolaire d'un savoir et d'un savoir-faire en matière de littérature.

Formalisme procédurier - dommageable pour la quête d'un sens humaniste; exception littéraire - et exclusion de la littérature de la sphère des expériences ordinaires; passivité des élèves - et inhibition de tout engagement individuel dans la lecture et l'interprétation collective des textes littéraires. Voilà trois diagnostics possibles des obstacles actuels à la relance de l'enseignement de la littérature. Ils sont très différents

4. Voir S. Terwagne, S. Vanhulle, A. Lafontaine, Les cercles de lecture. 
dans leurs présupposés et dans leurs implications. Mais tous consistent, en creux, à déplorer un héritage malheureux des études littéraires dans l'enseignement, les effets regrettables de ce que l'on pourrait appeler la transposition didactique de la nouvelle critique dans les classes.

L'exception littéraire coupe l'enseignement de la littérature d'autres pratiques de simulation virtuelle très familières et très appréciées de la plupart des adolescents, comme les jeux vidéo. Le formalisme, qui s'est constitué dans un refus de "l'illusion référentielle», vide les textes de toutes les prises existentielles auxquelles pourraient se raccrocher les élèves pour trouver quelque intérêt à leur lecture. Le dénigrement de la lecture naïve - la lecture dite "pour l'histoire» ou par identification aux personnages - a conduit à écarter comme une menace l'investissement spontané des élèves dans ce qu'ils lisent, c'est-à-dire les modalités ordinaires de réappropriation des formes de l'expérience exemplifiées dans les œuvres littéraires, coupant de ce fait court à tout processus scolaire d'empowerment interprétatif.

\section{Solipsisme de la lecture, communauté des lecteurs}

Pour ma part, j'ai une manière très simple de résumer cet état de fait. Il me semble en effet que les tropismes des études littéraires, depuis les années 1960 au moins, ont systématiquement privilégié le second degré de la littérature. Et que ce postulat implicite pose problème à l'école.

Par second degré, il faut entendre ceci: la valeur des textes littéraires a été assimilée, pour l'essentiel, à leur réflexivité critique - à la façon dont ils déconstruisent les autres discours, aux ruses linguistiques par lesquelles ils déjouent les normes de la langue, à leur statut de palimpseste intertextuel (faut-il rappeler que le sous-titre de l'ouvrage de Gérard Genette, Palimpsestes, est précisément: La littérature au second degré?), à leur effort de mise en abyme du geste littéraire lui-même et, dans un autre registre, plus axiologique, à une ironie vis-à-vis de tout énoncé ou tout discours qui prétendrait, par bêtise ou calcul manipulateur, dire quelque chose de crédible sur le monde.

L'immersion dans l'univers fictionnel, sensoriel ou symbolique de l'œuvre littéraire, de même que l'intérêt pour l'intrigue ou l'empathie pour les personnages, dans cette perspective, résulteraient d'une adhésion regrettable au faire-croire des ouvres. La référence est une illusion, le 
récit est un piège (comme le déclarait Louis Marin ${ }^{5}$ ), le personnage est une créature exclusivement textuelle (dont il convient seulement de décortiquer le trompe-l'œil): tous ces mots d'ordre ont rendu illégitime ce que j’appelle le premier degré de la littérature ${ }^{6}$.

Un premier degré, d'ailleurs, qui ne concerne pas seulement la lecture - investie affectivement et cognitivement - que l'on peut faire des textes, mais que j'étends, en outre, à une certaine puissance qu'ont les œuvres d'instituer des mondes dont on peut faire l'expérience - non pas tant des mondes possibles, car cela supposerait un monde actuel exempt de toute interférence avec la littérature ou la fiction, que des versions de la réalité, des institutions différenciées du réel offertes à ceux qui feraient le pari d'y croire pour de bon.

Premier et second degrés de la littérature ne désignent pas des strates du sens: le sens littéral, auquel s'attache la compréhension, ne résume pas le premier degré dont je parle ici; et, à vrai dire, les deux n'ont rien à voir, ou presque; par ailleurs, le sens figuré ou implicite, le montré (par opposition au dit), que traque une interprétation, ne doit pas être confondu avec le second degré. Le premier et le second degrés que je tâche de conceptualiser n'ont pas trait au sens des textes, mais à l'expérience que les œuvres programment et ouvrent au lecteur.

Le second degré rend possible une expérience réflexive et critique il appelle le sujet, le temps de la lecture, voire au-delà, à se déprendre de ses préjugés, de ses «idées reçues", de son sens commun, de ses illusions ordinaires, de tous les codes censés l'aliéner malgré lui, etc. C'est le régime éminemment égotiste de la souveraineté absolue du sujet.

Le premier degré, lorsqu'il n'est plus condamné à être l'illustration commode de tout ce qu'il ne faut pas faire quand on lit, désigne un espace d'expérience où le lecteur, dirait Thomas $\mathrm{Pavel}^{7}$, se met à l'écoute de la littérature, se livre sans défiance à l'univers d'une œuvre, s'en remet aux formes littéraires comme à des cadres d'expérimentation, s'expose à une pensée autre, à des affects insoupçonnés, à des calibrages inédits de la sensation et de la perception qui viendront peut-être enrichir, sinon modifier, le monde ordinaire qu'il tient pour vrai, intelligible

5. L. Marin, Le Récit est un piège.

6. Que l'on me permette de renvoyer, par commodité, à mon article: «Le premier degré de la littérature».

7. T. Pavel, Comment écouter la littérature? 
et indépassable. Le lecteur prend alors le risque d'être dupe, parce qu'il fait confiance à tel ou tel genre dont il partage les valeurs ou la schématisation, à tel écrivain ou à telle œuvre.

Ce faisant, le lecteur ne s'isole pas, comme ce serait le cas s'il s'en tenait exclusivement au second degré, dans un scepticisme radical qui lui ferait entrevoir toute dépendance et tout lien, fût-il d'amour ou d'amitié, comme une aliénation et une atteinte à son autonomie; il ne souscrit pas à une conception prométhéenne et solipsiste de l'émancipation. Il rejoint au contraire la communauté de celles et ceux qui croient ou ont cru aux mêmes schématisations littéraires du monde que lui - il participe d'une coalition discrète dont l'un des effets immédiats est de redéfinir ce que ses membres tiennent pour vrai, crucial et digne d'expérience. On pourrait, en suivant sur ce point Jacques Rancière, avancer qu'un tel lecteur entérine un "partage du sensible» engagé dans une œuvre ${ }^{8}$.

Cette philosophie de la littérature ne doit pas être confondue avec certaines des propositions de l'herméneutique. Ce n'est pas le sens des textes, mais l'usage des œuvres qui compte ici; la littérature n'est pas envisagée comme un accès privilégié au monde, qui en dévoilerait des aspects inconnus - elle institue au contraire des ontologies différenciées. Elle ne réfère pas, même indirectement, à une réalité qui lui préexisterait - elle pose, par ses référentialisations, des entités et des relations susceptibles de devenir réelles dans l'expérience des lecteurs.

Le premier degré de la littérature, ainsi conçu, a d'abord pour horizon le parti pris radical d'une relativité de l'ontologie, et la conviction démocratique d'une institution collective et égalitaire de ce dont on peut faire l'expérience à l'échelle humaine. De ce fait la littérature, entendue comme l'une des modalités possibles de cette institution des cadres de l'expérience, n'est plus assignée à la re-présentation d'une réalité qu'on décrète plus vraie et plus objective qu'elle; elle initie un processus de subjectivation des lecteurs et de cadrage de leurs possibles existentiels. Un tel processus est en outre collectif. Le lecteur n'est pas isolé face à son livre, avec la seule ressource de sa propre trajectoire biographique; il ne reçoit pas dans son coin le sens d'un texte littéraire. Il est face à son livre - dont les sollicitations, pour peu qu'il y réponde (et comment n'y répondrait-il aucunement, puisqu'il lit?), l'inscrivent d'emblée dans une communauté

8. J. Rancière, Le Partage du sensible. 
que fédère une adhésion à ce que l'œuvre, selon lui, tient pour sensible, intelligible ou croyable. Cette communauté virtuelle de lecteurs naît de l'assentiment accordé, le temps de la lecture au moins, à l'axiomatique de l'œuvre, c'est-à-dire à une granularité particulière du réel, à une abstraction spécifique de l'expérience et à une éthique formalisant aussi bien les rapports des lecteurs à ce qui est mis en scène (empathie, raillerie, indifférence, etc.) que les liens des lecteurs entre eux (communauté indistincte et spontanée des "belles âmes», choisie et hautaine des happy few ou, plus récemment, «désœuvrée», sinon «inavouable») .

\section{Initiation scolaire au premier degré de la littérature}

Trois diagnostics, une théorie de l'imagination littéraire: il reste maintenant à revenir aux considérations sur l'enseignement de la littérature, pour envisager pourquoi et comment ce premier degré de la littérature pourrait s'inviter dans les classes. Il convient donc de prendre les réflexions qui suivent comme une expérience de pensée, destinée à imaginer, dans ses grandes lignes, un futur éventuel de l'enseignement de la littérature.

Je m'appuierai à cet effet sur deux foyers très actifs de réflexion en didactique de la littérature: celui qui s'est constitué autour de la notion de «lecture littéraire», et celui que résume l'expression très débattue de «sujet lecteur ${ }^{10}$. Il me revient donc de répondre aux deux questions suivantes: la "lecture littéraire» peut-elle prendre en compte le premier degré, tel que je l'ai défini? Le "sujet lecteur» est-il similaire au lecteur dont je cherche pour ma part à décrire l'exposition aux ontologies littéraires?

La première chose qui frappe, dans ces deux notions, la «lecture littéraire» et le «sujet lecteur» - la lapalissade est ici inévitable -, c’est

9. Le lecteur que cette série brute d'assertions peu étayées rendrait curieux pourra se référer à certains autres de mes textes déjà publiés où il trouvera un raisonnement moins elliptique sur plusieurs de ces points (soit: «Ontologie letterarie dello spazio parigino»; "L'institution imaginaire de la fiction»; Balzac, une éthique de la description; "Une réalité à mi-hauteur").

Io. Voir notament J.-L. Dufays, L. Gemenne, D. Ledur, Pour une lecture littéraire 1.; J.-L. Dufays, "Les lectures littéraires»; G. Langlade, A. Rouxel (éds), Le sujet lecteur; B. Dunay, «Le sujet lecteur». 
précisément l'accent mis sur l'acte de lecture. L'héritage des théories de la réception est assumé dans ces travaux - et je rappelle ce truisme à la seule fin de prendre la mesure d'un tel consensus.

Que l'on y songe seulement: déléguer au seul acte de lecture l'autorité d'activer la dimension littéraire des textes - de trancher, autrement dit, la question de leur littérarité - implique au préalable d'avoir retiré cette autorité à toutes les autres instances qui pourraient y prétendre: à l'auteur, bien sûr, mais également à une certaine force inscrite dans la forme même des œuvres.

Laissons de côté l'intentionnalité auctoriale: elle soulève des questions qui nous embrouilleraient ici davantage que nécessaire. Prenons ce dernier point: l'autorité des œuvres elles-mêmes. Jean Rousset, qui nous accompagne le temps de cette réflexion, disait tout bonnement: "les intentions de l'œuvre " ${ }^{11}$. Rien n'est moins évident aujourd'hui. L'idée même déconcerte. Et pourtant, regardons-y de plus près: Le Misanthrope, Candide, Illusions perdues, Un coup de dés jamais n'abolira le hasard, Du côté de chez Swann, Nadja, En attendant Godot - voilà des configurations soigneusement organisées de signes que l'on peut soustraire à la juridiction de leur auteur, et auxquelles on peut de surcroît faire subir les variations cumulées de la philologie, de la génétique et de la bibliographie matérielle - elles n'en demeurent pas moins, sauf coup de théâtre éditorial imprévu, suffisamment stabilisées pour imposer à tout lecteur les caractéristiques intrinsèques de leur organisation.

On peut dire, bien sûr, que c'est le lecteur seul qui rend ces œuvres littéraires, du fait même qu'il les lit avec une attention qui en reconnaît la dimension esthétique. Mais on peut dire également, tout aussi justement me semble-t-il, que ces œuvres portent en elles, du fait de leur stabilisation, une force de reconnaissance esthétique qui se désigne comme telle à tout lecteur emporté par la logique interne de leur composition.

Cette idée n'exclut pas qu'il faille parfois se familiariser avec les manières spécifiques qu'ont ces œuvres de se présenter comme littéraires: autrement dit, il faut parfois être introduit aux différentes déclinaisons historiques notamment - du premier degré de la littérature pour pouvoir être emporté par les œuvres littéraires.

L'enjeu du premier degré de la littérature consiste donc, dans un premier temps, à apprendre aux élèves les façons dont les œuvres ou les

II. J. Rousset, Forme et signification, p. XVI. 
genres littéraires ont cherché ou cherchent à impliquer leurs lecteurs, à défaut de toujours y parvenir. Et cet apprentissage nécessite, de la part des élèves, qu'ils se plient aux vecteurs d'immersion, de catharsis ou de pathos inscrits dans les ouvres - qu'ils se soumettent, en ce sens, à l'autorité des formes qu'ils explorent, avant même d'y répondre individuellement en tant que lecteurs.

On comprend qu'il faille s'appuyer dans cette démarche sur des savoirs historiques, mais également narratologiques ou poétiques. On devine également ce qui nous manque encore en termes de ressources analytiques pour décrire adéquatement les diverses façons dont la littérature sollicite le lecteur, l'attire dans chacun de ses univers, le constitue en sujet d'une expérience esthétique et, surtout, lui propose une ontologie inédite, l'inscrit dans une communauté de lecteurs avant de prendre congé de lui après un nombre de pages fixé d'avance. Ces lacunes (savoir encore exploratoire, transposition didactique embryonnaire) rendent presque impossible, à l'heure actuelle, la transmission scolaire d'un savoir en la matière (raison de plus, je crois, pour s'y atteler sans retard).

Ce qui est en outre demandé à l'élève, dans une telle démarche d'apprentissage, n'engage en rien sa subjectivité, si l'on entend par là l'ensemble de ce qui tisse son intimité singulière. Le premier degré de la littérature privilégie et examine les processus littéraires de subjectivation. La réaction spontanée des élèves face à un texte peut être un embrayeur de l'apprentissage, à condition de ne pas perdre de vue ce qui nous occupe ici : le premier degré de la littérature s'attache aux cadres impersonnels de la subjectivation, et non à tel ou tel lecteur; il désigne les conditions sous lesquelles une œuvre littéraire offre à ses lecteurs un certain usage de leur imagination.

Deux éléments plaident donc, de ce point de vue, en faveur de l'introduction dans les classes d'une approche calquée sur le premier degré de la littérature: (i) le premier élément tient à la conceptualisation de l'œuvre littéraire comme matrice impersonnelle de subjectivation, endeçà donc, ou en amont, de toute appropriation individuelle et de tout appel à l'intimité des élèves dans le cadre de la classe ${ }^{12}$; (ii) le second

I2. Intimité des élèves sur laquelle l'institution scolaire prendrait le risque de statuer de façon normative si elle la mobilisait comme telle dans les processus d'apprentissage (voir sur ce point K. Canvat, "Apprentissage de la lecture et enseignement de la littérature»). 
élément tient au rappel de cette communauté de lecteurs impliqués que l'œuvre littéraire cherche à instituer, avec ce que cela ouvre de possibilités à la classe, comme collectif, de répondre ou non à ces liens particuliers que la littérature instaure souvent entre les lecteurs.

Dans un second temps, l'apprentissage du premier degré de la littérature peut inclure un moment interprétatif de retour sur les cadres de subjectivation engagés dans les œuvres étudiées en classe: à quelles ressources formelles sont-ils associés? En repère-t-on plusieurs entre lesquels le lecteur est sommé de choisir? Qu’implique ce choix? La plongée dans l'univers de l'œuvre induit-elle un usage de l'imagination qui orienterait le lecteur vers un deuxième degré de la littérature plus critique, plus réflexif ou plus intertextuel? Ces cadres, s'ils sont anciens, trouventils des échos dans les diverses façons dont les adolescents (à qui s'adresse l'enseignement) s'envisagent eux-mêmes aujourd'hui ? etc.

Sous réserve de ces aménagements (sans doute moins importants qu'il n'y paraît), mon questionnement rejoint, je crois, les interrogations en termes de "lecture littéraire" et de "sujet lecteur». Et ce que j’ai dit des pistes à suivre pour imaginer un renouvellement de l'enseignement littéraire croise certaines gammes d'activités déjà proposées par les didacticiens.

Par contre, la perspective du premier degré de la littérature promet sans doute à ces interrogations et à ces activités des développements que les références à la nouvelle critique ou à la théorie de la réception rendaient jusqu'alors louches, ou tout simplement impensables.

Jérôme DAvid Université de Genève 


\section{BIBLIOGRAPHIE}

Bourdieu, Pierre, Passeron, Jean-Claude, Les Héritiers. Les étudiants et la culture, Paris, Minuit, 1964.

—, La Reproduction. Eléments pour une théorie du système d'enseignement, Paris, Minuit, 1970.

Canvat, Karl, "Apprentissage de la lecture et enseignement de la littérature. Enjeux, finalités, articulations", in Lecture privée et lecture scolaire. La question de la littérature à l'école, sous la dir. de Patrick Demongin, Jean-François Massol, Centre régional de documentation de l'académie de Grenoble, 1999, p. 15-28.

Citton, Yves, Lire, interpréter, actualiser. Pourquoi les études littéraires?, Paris, Editions Amsterdam, 2007.

Daunay, Bertrand, «Le sujet lecteur: une question pour la didactique du français ", Le français aujourd' hui, 157 (2007), p. 43-51-

DAVID, Jérôme, "Ontologie letterarie dello spazio parigino : romiti e "flâneurs" del primo ottocento", Quaderni Storici, 125 (2007), p. $433-460$.

—, "L'institution imaginaire de la fiction", in Devant la fiction, dans le monde, sous la dir. de Catherine Grall, Marielle Macé, Rennes, Presses Universitaires de Rennes, 2010, p. 177-194.

—, Balzac, une éthique de la description, Paris, Champion, 2010.

—, "Une réalité à mi-hauteur: exemplarités littéraires et généralisations savantes au XIX ${ }^{\mathrm{e}}$ siècle", Annales. Histoire, sciences sociales, 2 (2010), p. 263-290.

—, «Le premier degré de la littérature», Fabula-LhT, 9 (2011), http:// www.fabula.org/lht/9/david.html, page consultée le 28 octobre 2013.

Dufays, Jean-Louis, Gemenne, Louis, Ledur, Dominique, Pour une lecture littéraire 1. Approches historiques et théoriques, Bruxelles, De Bœck, 1996.

Dufays, Jean-Louis, "Les lectures littéraires: évolution et enjeux d'un concept», Tréma, 19 (2002), p. 5-16. 
Langlade, Géradr, Rouxel, Annie (éds), Le sujet lecteur. Lecture subjective et enseignement de la littérature, Rennes, Presses Universitaires de Rennes, 2004.

Marin, Louis, Le Récit est un piège, Paris, Minuit, 1978.

Pavel, Thomas, Comment écouter la littérature?, Paris, Fayard, 2006.

Rancière, Jacques, Le Partage du sensible, Paris, La Fabrique, 2000.

Rousset, Jean, Forme et signification. Essais sur les structures littéraires de Corneille à Claudel, Paris, José Corti, 1986 (1962).

Schaeffer, Jean-Marie, Petite écologie des études littéraires. Pourquoi et comment étudier la littérature?, Vincennes, Editions Thierry Marchaisse, 2011.

Terwagne, Serge, Vanhulle, Sabine, Lafontaine, Annette, Les cercles de lecture: interagir pour développer ensemble des compétences de lecteurs, Bruxelles, De Boeck, 2007 (3 édition).

Todorov, Tzvetan, La littérature en péril, Paris, Flammarion, 2007. 